\title{
Rise of the second order
}

\section{$C 4$ \\ OD topological corner states could form the basis for a new generation of highly reliable data-storage and processing devices}

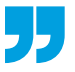

Topological insulators are insulating in the bulk but host topologically protected conductive states at their edges. This materials family is continuously expanding with new members: among the latest are higher-order topological insulators, which host topologically protected states at the edges of their edges. For example, a 2D sample hosting $0 \mathrm{D}$ topological corner states is a second-order topological insulator (SOTI). SOTIs have been realized in various linear systems, including electronic, microwave and acoustic platforms. Now Farzad Zangeneh and Romain Fleury, writing in Physical Review Letters, show that introducing nonlinearities can lead to SOTIs with self-induced corner states that can be reconfigured using an external control knob.

Topologically protected corner states are attractive for applications because they can concentrate the energy of the system at points where it can be harvested. "Our environment is full of wasted energy that

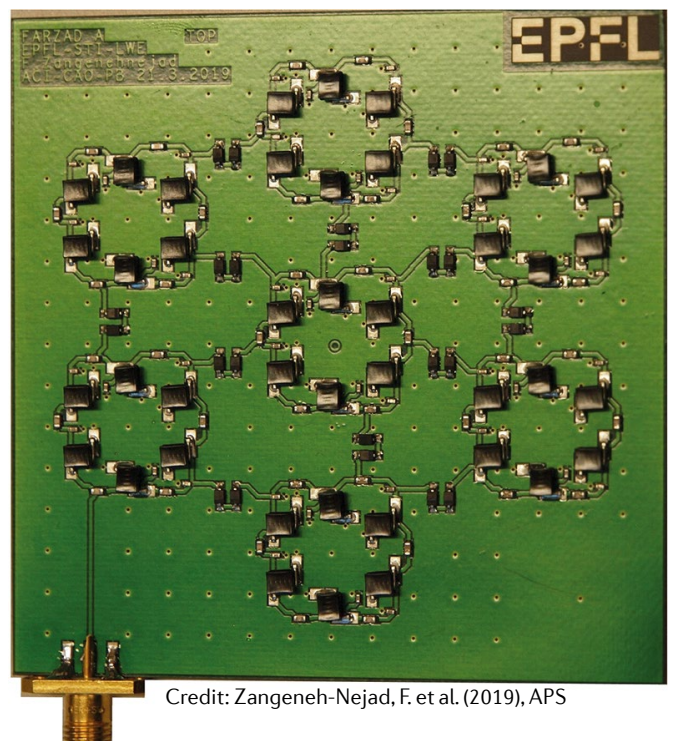

we would like to re-use, from the vibrations of building walls and roads to water waves or even unused electromagnetic energy from our Wi-Fi networks," notes Fleury. "But manipulating and focusing a big amount of wave energy diluted over a large volume is a challenging problem." SOTIs can offer a solution, because when they are loaded with a sufficient amount of wave energy they send it all to their corners. For mechanical waves, for instance, we can imagine a carefully structured wall that would send all vibrations to its corners, where a small number of energy harvesters could be placed. "The problem is that SOTIs cannot be directly applied to focusing diluted energy distributions, because they are based on insulating materials that, by definition, can store very little energy in their bulk," explains Fleury. "To circumvent this issue, we proposed the concept of non-linear SOTIs: we start from a conventional material and load its bulk with a certain amount of energy, then by judiciously exploiting non-linear effects we can induce a selfassembly of the energy distribution at the corners."

The authors tested the feasibility of their idea by studying a tightbinding lattice composed of evanescently coupled resonators arranged in a hexagonal pattern. The resonators are linearly coupled within each cell, whereas neighbouring cells are non-linearly coupled with one another. A sufficiently high excitation intensity can harness the inherent non-linearities of the system, pushing it into a topological phase. As the intensity increases, two types of topological state emerge: ordinary helical edge modes and second-order 0D corner modes. The higher the intensity, the more localized the topological modes.

The realization of the proposed model in a nonlinear electronic circuit lattice - in which the part of the atoms was played by resonant circuits and that of the nonlinear connections by diodes - proved the validity of the idea and led to the observation of the expected corner states. "For the purpose of concept demonstration we wanted to use a simple, relatively inexpensive platform that would fit on a tabletop and be familiar to most people, including our students," says Zangeneh. "We therefore decided to build a prototype using an electronic circuit, which allows an easy implementation of both resonators (via inductors and capacitors) and non-linearities (via electronic diodes), the key ingredients of our theory."

Because the proposed approach is very general, similar self-induced and reconfigurable SOTIs can most likely be obtained in photonic, phononic and microwave systems, with huge potential for applications. " $0 \mathrm{D}$ topological corner states could form the basis for a new generation of highly reliable data-storage and processing devices," comments Fleury. "The strong confinement of the corner states also represents an opportunity in sensing and, in particular, bio-sensing applications, with the added benefit that the characteristics of the sensor would be externally adjustable."

Giulia Pacchioni

ORIGINAL ARTICLE Zangeneh-Nejad, F. \& Fleury, R. Nonlinear second-order topological insulators. Phys. Rev. Lett. 123, 053902 (2019) 